\title{
Diabetes and risk of frailty and its potential mechanisms: a prospective cohort study of older adults.
}

\section{Short title: Diabetes and frailty}

Authors: Esther García-Esquinas $(\mathrm{PhD})^{1 \dagger}$, Auxiliadora Graciani $(\mathrm{PhD})^{1}$, Pilar Guallar-

Castillón $(\mathrm{PhD})^{1}$, Esther López-García $(\mathrm{PhD})^{1}$, Leocadio Rodríguez-Mañas $(\mathrm{PhD})^{2}$, Fernando Rodríguez-Artalejo $(\mathrm{PhD})^{1}$

Affiliations: ${ }^{1}$ Departamento de Medicina Preventiva y Salud Pública, Universidad Autónoma de Madrid / IdiPaz, and CIBER of Epidemiology and Public Health (CIBERESP), Madrid, Spain.

${ }^{2}$ Division of Geriatric Medicine, Hospital Universitario de Getafe, Madrid, Spain

\section{†Corresponding author:}

Esther García García-Esquinas, MD, PhD

Department of Preventive Medicine and Public Health, School of Medicine.

Universidad Autónoma de Madrid.

Calle del Arzobispo Morcillo 4.

28029 Madrid, Spain

Phone: (+34) 91-497-27-61

E-mail: esthergge@gmail.com

Number of words (text), number of words (abstract), number of tables and figures Abstract word count: 267

Word count: Introduction through conclusions: 3566

Number of tables: 4

Number of figures: 0 


\section{List of abbreviations:}

BMI: Body Mass Index

Hs-CRP: High-sensitivity C-reactive protein

HbA1c: Glycated hemoglobin

HDL: High-density lipoprotein

LDL: Low-density lipoprotein

Keywords: Diabetes Mellitus, frailty, walking speed, older adults, Spain. 


\section{ABSTRACT}

Background: There is emerging evidence of the role of diabetes as a risk factor for frailty. However, the mechanisms of this association are uncertain.

Methods: Prospective cohort study of 1750 non-institutionalized individuals aged $\geq 60$ years recruited in 2008-2010. At baseline, information was obtained on health behaviors, morbidity, cardiometabolic biomarkers, and antidiabetic treatments. Individuals were considered diabetic if they reported a physician-diagnosis or had fasting serum glucose $\geq 126 \mathrm{mg} / \mathrm{dl}$. Study participants were followed-up through 2012 to assess incident frailty, defined as at least three of the five Fried criteria.

Results: At baseline, the cohort included 346 individuals with diabetes and 1404 without it. Over a mean 3.5-year follow-up, 115 cases of incident frailty were ascertained. After adjustment for age, sex and education, participants with diabetes showed an increased risk of frailty (odds ratio [OR]: 2.18; $95 \%$ confidence interval [CI]: 1.42-3.37). Additional adjustment for health behaviors and abdominal obesity yielded a $29.7 \%$ reduction in the OR (OR: 1.83; 95\%CI: 1.16-2.90). Subsequent adjustment for morbidity produced an additional $8.4 \%$ reduction (OR: 1.76; 95\%CI: 1.10-2.82), and for cardiometabolic biomarkers, a further 44\% reduction (OR: 1.32; 95\% 0.70-2.49). In particular, adjustment for HbA1c, lipoproteins and triglycerides accounted for the greatest reductions. Finally, additional adjustment for oral antidiabetic medication reduced the OR to 1.01 (95\%CI: 0.46-2.20), while adjustment for nutritional therapy increased it to 1.64 (95\%CI: $0.77-3.49)$.

Conclusions: Diabetes mellitus is associated with higher risk of frailty; this association is partly explained by unhealthy behaviors and obesity and, to a greater extent, by poor glucose control and altered serum lipid profile among diabetic individuals. Conversely, diabetes nutritional therapy reduces the risk of frailty. 


\section{INTRODUCTION}

In 2013, the worldwide prevalence of diabetes mellitus among those aged 60 to 79 years was around $19 \%$, accounting for over $35 \%$ of all adult cases of diabetes and around $70 \%$ of the global health expenditure for the disease (1). According to projections from the International Diabetes Foundation (IDF), by 2035 the number of patients with diabetes will have risen to 590 million (1). The largest increase is projected among the elderly, with an expected 252.8 million cases in 2035 .

Diabetes is one of the leading causes of disease burden in old age (2). Diabetic older adults are at increased risk of cardiovascular disease and cancer, as well as cognitive dysfunction (3), functional limitations and disability (4,5). Frailty, a geriatric syndrome characterized by declines in functioning across multiple systems, is also an important predictor of disability (6). However, very few studies have evaluated the prospective relationship between diabetes and frailty (7-10), and the mechanisms that could explain this association are uncertain.

In this study, we hypothesize that three types of factors could help explain the potential association between diabetes and frailty. First, it is well known that diabetes is associated with unhealthy lifestyles (e.g., poor diet, sedentary behavior) and severe morbidity (e.g., obesity, cardiovascular disease) (1), which in turn may lead to frailty (11). Second, certain cardio-metabolic abnormalities frequently found in diabetes (e.g., chronic inflammation, hyperglycemia, dyslipidemia or high blood pressure), which are mediators of some of its microvascular and macrovascular complications, could also exert a role in frailty development. Third, there is evidence that the prognosis of diabetic patients can be improved by nutrition and glucose-lowering therapy, as well as by

appropriate management of cardiovascular risk factors (hypertension and 
hyperlipidemia) (12), and we hypothesize that these treatments could also reduce the risk of frailty among diabetic older adults.

Accordingly, this study has two objectives. First, we evaluated the prospective association between diabetes and the risk of frailty in older adults. Second, we examined the degree to which this association could be explained by unhealthy behaviors, comorbid conditions and cardio-metabolic biomarkers associated with diabetes, as well as the role of antidiabetic treatment and antihypertensive or lipidlowering medication.

\section{PARTICIPANTS AND METHODS}

\section{Study participants and design}

We analyzed data from the Seniors-ENRICA cohort, whose methods have been reported elsewhere (13). This cohort was established in 2008-2010 with 2614 noninstitutionalized individuals aged $\geq 60$ years. At baseline, computer-assisted telephone interviews were used to obtain information on socio-demographic factors, health behaviors and morbidity. Additionally, home visits were performed to conduct a physical examination, obtain 12-h fasting blood samples and record usual diet and prescribed medication. Participants were followed-up until 2012, when a second wave of data collection was performed, again including a phone interview, a physical exam, and diet and medication assessment at home. Ninety-five participants (3.6\%) died during follow-up. From the remaining 2519 subjects, 2085 had complete information on frailty at the end of follow-up. From these 2085 individuals, we further excluded 174 who either lacked information on frailty or were frail at baseline, 36 without complete data to evaluate their diabetic status and 135 with missing information on other study variables, leading to a final sample of 1750 individuals. 
All study participants gave written informed consent, and the Clinical Research Ethics Committee of the 'La Paz' University Hospital in Madrid approved the study.

\section{Study variables}

\section{Frailty}

Frailty was assessed at baseline and at the end of follow-up with a slight modification of the phenotypic criteria proposed by Fried et al. in the Cardiovascular Health Study (CHS) (14). Individuals meeting $\geq 3$ of the following five criteria were considered as frail: 1) Self-reported exhaustion, based on a response of " $\geq 3 / 4$ days a week" to any of the following questions from the Center for Epidemiologic Studies Depression Scale : "I felt that anything I did was a big effort" or "I felt that I could not keep on doing things"; 2) Weight loss, defined as unintentional loss of $\geq 4.5 \mathrm{~kg}$ of body weight in the preceding year; 3) Low physical activity, defined as walking $\leq 2.5 \mathrm{~h} /$ week in men and $\leq 2$ $\mathrm{h}$ /week in women; 4) Weakness, defined as the lowest quintile in the CHS of maximum strength in the dominant hand adjusted for sex and body mass index. Strength was measured as the highest of two consecutive measures with a Jamar dynamometer; and 5) Slow walking speed, defined as the worst cohort-specific quintile in the three-meter walking speed test, adjusted for sex and height.

\section{Diabetes mellitus}

Participants were considered diabetic if they reported a physician-diagnosis of diabetes or had fasting serum glucose $\geq 126 \mathrm{mg} / \mathrm{dl}$. Glucose was measured using the oxidase glucose technique (ADVIS 2400 Chemistry System analyzer, Siemens).

Sociodemographic variables, lifestyle, obesity and reported comorbidity 
At baseline, self-reported information was obtained on age, sex educational level, tobacco and alcohol consumption. Physical activity was measured with the questionnaire used in the EPIC-Spain cohort (15), and sedentary behavior was estimated by time spent watching television as assessed by the Nurse's Health Study questionnaire validated in Spain (16). Food consumption in the previous year was collected using a validated computerized diet history developed from that used in the EPIC-Spain cohort study, and energy intake was calculated using Spanish food composition tables (17). Adherence to the Mediterranean diet was summarized using the Mediterranean Diet Adherence Screener (MEDAS) index (18).

Baseline weight, height and waist circumference (WC) were measured at home. Measurements were performed twice using electronic scales (model Seca 841, precision to $0.1 \mathrm{~kg}$ ), portable extendable stadiometers (model Ka We $44444 \mathrm{Seca}$ ) and flexible, inelastic belt-type tapes, respectively. Mean values of the two measurements were used for the analyses. Body Mass Index (BMI) was calculated as weight in kg divided by square height in $\mathrm{m}$. Obesity was defined as BMI $\geq 30 \mathrm{~kg} / \mathrm{m}^{2}$, and abdominal obesity as WC $>102 \mathrm{~cm}$ in men and $>88 \mathrm{~cm}$ in women.

Participants also reported if they had previously suffered from any of the following physician-diagnosed diseases: cardiovascular disease (ischemic heart disease, stroke or heart failure), cancer, chronic respiratory disease (chronic bronchitis or asthma), osteoarthritis, arthritis or hip fracture.

\section{Cardio-metabolic biomarkers}

High sensitivity C-reactive protein (hs-CRP) was determined by latex-enhanced nephelometry. Fibrinogen was measured by the coagulation method and leptin by enzyme-linked immunoassay (DBC, Diagnosis Biochem Canada, Inc). Triglycerides 
were measured by the glycerol phosphate oxidase method; total cholesterol by enzymatic methods using cholesterol esterase and cholesterol oxidase; HDL cholesterol by the direct method using elimination/catalase; and LDL cholesterol was estimated using the Friedewald formula..Glycemic control was assessed by the level of glycated hemoglobin (HbA1c), measured by high-performance liquid chromatography (Adams A1c HA-8160, Arkray).

Blood pressure was measured with standardized procedures using validated automatic devices (Omron model M6) and three cuff sizes according to arm circumference. Two sets of blood pressure readings were made separated by 90 minutes. In each set, blood pressure was measured 3 times at 1-2 minute intervals, after resting 3 to 5 minutes in a seated position. Systolic blood pressure was calculated as the mean of $\geq 3$ of the last 5 readings.

\section{Management of diabetes and cardiovascular risk factors}

Nutrition therapy was self-reported. Drug treatment for diabetes (including oral antidiabetics and insulin), hypertension and hyperlipidemia was checked by the study staff against drug packages at home.

\section{Other variables}

Functional limitations in instrumental activities of daily living (IADL) were assessed with the Lawton and Brody Scale. Due to cultural issues, the questions on subjects' ability to prepare meals, do household chores, and care for clothing were excluded in men. The presence of limitation in at least one IADL was considered as disability.

\section{Statistical analysis}


In a first step, we used logistic regression models to evaluate the association of lifestyle, morbidity, cardio-metabolic biomarkers, and management of diabetes and other cardiovascular risk factors at baseline, with risk of frailty. These models were adjusted for age, sex and educational level.

We then estimated the association between diabetes at baseline and risk of frailty using logistic regression models with progressive levels of adjustment. In the basic model, we only considered age, sex and educational level as covariates. We then fitted four models by progressively adding subsets of covariates according to the following pattern: 1) Lifestyle (smoking, alcohol consumption, time spent watching television, physical activity, MEDAS score, energy intake) and anthropometric factors (either abdominal or general obesity); 2) Morbidity (cardiovascular disease, chronic respiratory disease, cancer, arthritis, osteoarthritis, fracture) ; 3) Cardio-metabolic biomarkers (CRP, fibrinogen, leptin, systolic blood pressure, triglycerides, HDL-cholesterol, LDLcholesterol, HbA1c); and 4) Management of diabetes and cardiovascular risk factors (diabetes, nutritional therapy, oral antidiabetics, insulin, antihypertensive or lipidlowering medication).

Next, we estimated the association between diabetes and onset of each frailty criterion among robust adults (free of all five criteria) at baseline. Again, four main models that accounted for the previously defined subsets of covariates were fitted.

Statistical significance was set at two-sided $\mathrm{P}<0.05$. The analyses were performed with Stata ${ }^{\circledR}$, version 13.0.

\section{RESULTS}

Table 1 shows the socio-demographic, lifestyle, clinical and biochemical variables of the study participants, according to diabetic status. Compared to the non-diabetic 
individuals, those with diabetes were on average one year older, were more frequently men, had lower educational level and showed a higher prevalence of tobacco consumption, obesity and sedentary behavior. Also, they were less frequently moderate drinkers, showed a higher frequency of cardiovascular disease, a lower level of HDLand LDL-cholesterol and a higher level of triglycerides. Among diabetic participants, $65 \%$ were following nutritional therapy, and 50\% were being treated with oral antidiabetics and $12 \%$ with insulin.

During a mean 3.5-year follow-up, 115 cases of incident frailty were ascertained. Table 2 presents the results of logistic regression models examining the relationship between the main baseline variables and the risk of frailty. Older participants, women, participants with sedentary behavior or obesity, as well as those with osteoarthritis or arthritis showed an increased risk of frailty. Conversely, participants with higher educational level, moderate drinkers and those with higher adherence to the Mediterranean diet, showed a decreased risk of frailty. Regarding the cardio-metabolic biomarkers, a positive link with frailty was observed for CRP, leptin, tryglicerides and HbA1c, while a negative link was observed for HDL- and LDL-cholesterol.

During the follow-up, 76 individuals developed frailty among the 1404 non-diabetic subjects, and 39 among the 346 subjects with diabetes. Table 3 presents the main results of the study. After adjustment for age, sex and educational level, participants with diabetes showed an increased risk of frailty (odds ratio [OR]: 2.18; 95\% confidence interval [CI]: 1.42-3.37; Basic model). Additional inclusion of lifestyle factors and central obesity yielded a 29.7\% change in the OR (OR: 1.83; 95\%CI: 1.16-2.90; Model 1). This last result was virtually the same when considering general (BMI) instead of abdominal obesity. Further adjustment for morbidity at baseline (model 2) produced an additional $8.4 \%$ reduction in the OR (OR: 1.76; 95\%CI: 1.10-2.82), while adjustment 
for the cardio-metabolic biomarkeres (model 3) yielded an additional $44 \%$ reduction in the OR (OR: 1.32; 95\% 0.70-2.49), which lost significance. In particular, inclusion of HbA1c, lipoproteins and triglycerides accounted for the greatest reductions. Finally, while further adjustment of model 3 for insulin treatment, antihypertensive or lipidlowering medication resulted in a marginal change in the OR, adjustment for oral antidiabetics reduced the OR to 1.01 , and adjustment for nutritional therapy increased it to 1.64 .

To assess the robustness of the results, we conducted several sensitivity analyses. First, we replicated the analyses after excluding individuals with limitations in IADL. Second, we further adjusted the models for incident morbidity. And third, we repeated the analyses using the lowest quintile in our study sample for maximum strength in the dominant hand adjusted for sex and BMI. All these analyses yielded similar results to those presented in the main tables (data not shown).

Table 4 shows the effect of diabetes on each component of the frailty syndrome. The results suggest that the increased risk of frailty observed in individuals with diabetes is mainly driven by a reduction in walking speed and an increased risk of unintentional weight loss. Similar to the case for overall frailty, these associations were partly explained by the cardio-metabolic biomarkers (specifically higher HbA1c and worse lipid profile ) and were modulated by management of diabetes, in particular nutrition therapy. Diabetes also showed a non-statistically significant increased risk of muscle weakness. However, no association was observed with the risk of exhaustion or low physical activity.

\section{DISCUSSION}


In this study of community-dwelling older adults in Spain, diabetes was associated with an increased risk of frailty over 3.5 years of follow-up. This increased risk was partly explained by unhealthy behaviors and obesity and, to a greater extent, by poor glucose control and altered serum lipid profile among diabetic individuals. Conversely, nutritional therapy modulated the increased risk of frailty observed among subjects with diabetes.

Patients with type- 2 diabetes have an increased prevalence of lipid abnormalities, which contribute to their higher risk of cardiovascular events. As an example, results from the Multiple Risk Factor Intervention Trial, with 5,163 diabetic men and 342,815 nondiabetic men followed for an average of 12 years, showed that the absolute risk of coronary mortality for each $20 \mathrm{mg} / \mathrm{dl}$ increase in total cholesterol was 3-5 times higher in diabetic than in non-diabetic men (19). Similarly, poor glycemic control is known to increase the risk of long-term complications among diabetic individuals $(20,21)$, with evidence showing that a $1 \%$ point elevation in $\mathrm{HbA} 1 \mathrm{c}$ concentrations in persons with type 2 diabetes is associated with an approximate $18 \%$ increased risk of cardiovascular disease (20) and a 15\% increased risk of all-cause mortality (20). Our results suggest that poor glycemic and lipid control may also raise the risk of frailty associated with diabetes mellitus in older adults.

High concentrations of glucose might lead to systemic chronic inflammation, which has been identified as a risk factor for frailty (22). Some studies have also shown that older diabetic individuals have an accelerated loss of skeletal muscle strength $(23,24)$, which is more pronounced among those with poor glycemic control (HbA1c>8\%) (25). Still, to our knowledge only one previous study has evaluated the role of hyperglycemia in frailty development. Results from this investigation, based on the Women's Health and Aging Studies I and II, displayed a positive association between HbAlc and prevalent 
frailty, which was independent of coronary artery disease (26). Additionally, there is evidence from the Active Steps for Diabetes program that diabetes education can be effective in reducing $\mathrm{HbA} 1 \mathrm{c}$ and frailty (27).

Lower serum total cholesterol levels among older adults are predictive of all-cause mortality $(28,29)$ and functional decline (30). In the Three-City cohort study, with 6,141 participants aged $\geq 65$ years and followed up for 7 years, high triglycerides $(\geq 1.7$ $\mathrm{mmol} / \mathrm{L}$ ) were significantly associated with the incidence of mobility limitations (using the Rosow and Breslau scale) and with worse scores in the basic and instrumental activities of daily living scales (31). Also, low HDL-cholesterol concentrations $(\leq 1.04$ $\mathrm{mmol} / \mathrm{L}$ in men and $\leq 1.29 \mathrm{mmol} / \mathrm{L}$ in women) were associated with an increased risk of mobility limitations (31). Results from the ilSIRENTE study found that, in older adults aged $\geq 80$ years, HDL-cholesterol concentrations were inversely associated with physical function (as measured using a 4 meter walking-speed test, and the basic and instrumental activities of daily living scales) (32); and in this same study, frail individuals with high HDL-cholesterol levels at baseline showed better survival at two years (33).

In our study, receiving nutritional therapy decreased the diabetes-related risk of frailty. Evidence from randomized controlled trials shows that diabetes nutrition therapy is effective to improve blood glucose control (34). Recent trials have also indicated that evidence-based nutritional care is beneficial in reducing total triglycerides, cholesterol (35) and LDL levels (35) and increasing HDL-cholesterol (36). Often the initiation of nutritional therapy is accompanied by other lifestyle changes that can modulate the risk of frailty, including regular exercise or smoking cessation. Moreover, nutritional therapy can also produce weight loss, which has been shown to improve physical function among older adults, independently of changes in physical activity (37). 
In our study, treatment with oral antidiabetics seemed to aggravate the risk of frailty observed among diabetic subjects, an unexpected finding that was independent of glycemic control. Unfortunately, we were unable to evaluate the role of specific oral glucose-lowering agents on the association between diabetes and frailty. It has been hypothesized that the use of certain antidiabetic drugs (e.g, glibenclamide) could contribute to the age-related muscle atrophy and sarcopenia associated with type 2 diabetes (38), and there is also evidence that some oral antidiabetics (e.g, metformin or disaccharidase inhibitors) tend to promote weight loss (37). On the other hand, recent evidence from the Study of Osteoporotic Fractures suggested that use of insulin sensitizers (metformin and/or thazolidinedione) could attenuate the loss of usual walk speed observed in women with diabetes (39). In light of these conflicting data, future research should further evaluate the role of specific hypoglycemic agents on muscle weakness, functional impairment and risk of frailty.

Our results suggest that the increased risk of frailty observed among diabetic individuals is mainly driven by reductions in walking speed and, to a lesser extent, by an increased risk of unintentional weight loss and weakness.

Lower-limb muscle strength has been shown to be a good predictor of incident mobility limitations (40), hospitalization (41) and death (42) in older adults. In this regard, previous studies linking diabetes with reduced walking speed $(39,43-45)$ have attributed this association to the accelerated loss of skeletal muscle strength that is commonly seen in older adults with this metabolic disease $(23,24)$. To address whether diabetes-related muscle impairments could mediate the association between diabetes and low walking speed, researchers from the InCHIANTI study assessed walking performance and lower-extremity muscle mass, strength and power in a sample of 835 older adults (43). They found that lower-limb muscle characteristics accounted for $24.3 \%$ and $15.1 \%$ of 
walking speed difference comparing diabetic and non-diabetic subjects in a 4- and 400meter walk test, respectively. The Study of Osteoporotic Fractures (39) showed that diabetes was prospectively associated with declines in walking speed, but not in handgrip strength. This last finding is in accordance with the Health, Aging, and Body Composition Study (23), where, although type 2 diabetes was associated with an accelerated loss of leg muscle strength and quality, no association with arm muscle strength or quality was observed. Similarly, although in our study diabetes was associated with decreased walking speed, we observed no significant association between diabetes and hand-grip weakness. In addition to loss of lower-limb strength, the following mechanisms have been proposed as potential contributors to the decline in walking speed observed among diabetic individuals: poor sensory and motor peripheral nerve function (46), lower cerebral vasoreactivity (47), poor balance(46) and fear of falls (48).

Unintentional weight loss among diabetic individuals may occur due to uncontrolled hyperglycemia, use of certain oral anti-diabetics (e.g, sodium glucose transporter inhibitors) or coexistence of comorbid conditions (e.g, depression). In older persons, uncontrolled weight loss is often accompanied by decreases in muscle and bone mass, which can accelerate functional declines $(49,50)$ and in turn may increase the risk of frailty. In this sense, as occurs in the elderly from the general population (51), unintentional weight loss among diabetic adults has shown to be an important predictor of mortality (52).

Among the patients with diabetes in our study, the levels of HbA1c were quite low. In a previous work comparing the achievement of individualized glycemic target between Spain and the US, we found that Spanish diabetic patients over 45 years had better glucose control than those in the US, particularly among patients free of diabetes 
complications (53). These findings could be due to differences in comorbidities or in time since diagnosis between patients in both countries, and could also result from differences in access to health services and a greater overtreatment of glucose levels in Spain than in the US (54). Future research should address these differences in detail.

Strengths of this study include its prospective design and objective assessment of diabetes and frailty according to standard criteria. Also, we evaluated an extensive list of health behaviors, biomarkers and clinical variables that could contribute to the association between diabetes and frailty. Moreover, our results were robust to different sensitivity analyses. Among the potential limitations, no information was included about type of diabetes, although in elderly people type- 2 diabetes is predominant. Second, because we lacked information on postprandial serum glucose concentrations, we may have missed some patients with diabetes since fasting glucose can be below $126 \mathrm{mg} / \mathrm{dl}$ in the early stages, but have postprandial values above $200 \mathrm{mg} / \mathrm{dl}$. Third, we lacked complete information on specific antidiabetic agents. Fourth, information on comorbidities was self-reported so it is subject to recall bias. However, in Spain, a country with a good access to health services, which are free of charge, self- reporting of severe comorbidities is generally valid when compared to clinical records. Fifth, when evaluating functional limitations in IADL, exclusion of household chores, meal preparation and care of clothing tasks in men may have led to a small underestimation of disability in this gender group. Finally, we could not evaluate the contribution of lower extremity muscle properties to the observed associations.

In conclusion, diabetes mellitus is associated with a higher risk of frailty among noninstitutionalized older adults. A number of health behaviors and cardio-metabolic biomarkers partly explain this association; moreover, nutritional therapy may reduce the risk of frailty associated with diabetes. Future research should evaluate whether diet- 
and other behavioral interventions can reduce the risk of frailty among diabetic older adults. The role of specific antidiabetic agents in frailty should also be further investigated.

\section{LIST OF CONTRIBUTORS}

EGE and FRA conceived the study; AG, PGC, ELG researched data; EGE performed the statistical analyses; EGE and FRA drafted the manuscript; all authors reviewed the manuscript for important intellectual content; EGE and FRA had primary responsibility for final content. All authors read and approved the final manuscript. 
Table 1: Baseline characteristics of participants in the Seniors-ENRICA cohort study by diabetic status. $\mathbf{N}=\mathbf{1 7 5 0}$.

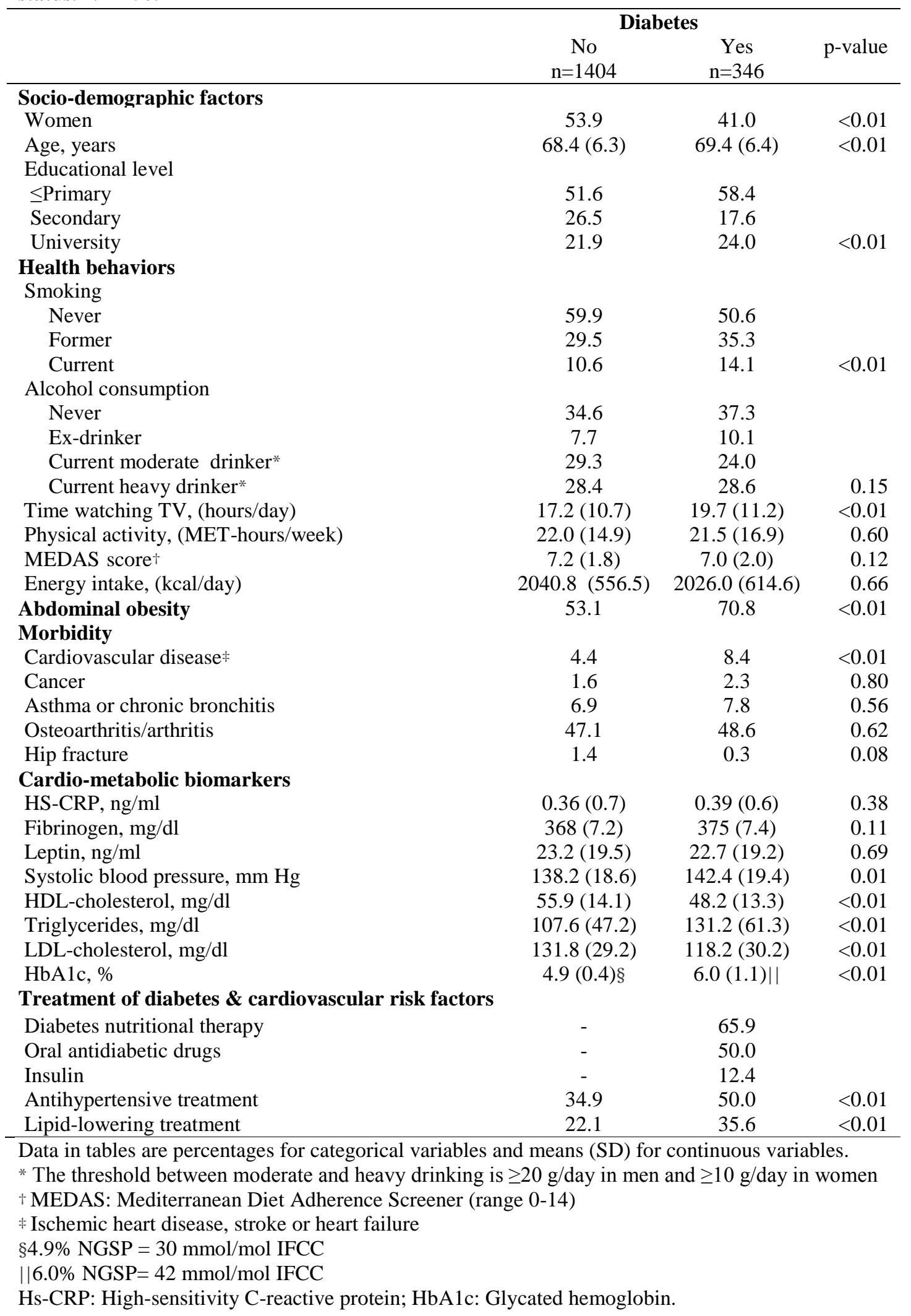


Table 2: Odds ratios (95\% confidence interval) of frailty by socio-demographic factors, health behaviors, clinical variables and cardio-metabolic biomarkers. All models are adjusted for sex, age and educational level.

\begin{tabular}{|c|c|}
\hline & $\begin{array}{c}\text { Odds ratio } \\
\text { (95\% confidence interval) }\end{array}$ \\
\hline \multicolumn{2}{|l|}{ Socio-demographic factors } \\
\hline Sex (women) & $2.50(1.61-3.90)$ \\
\hline Age, years & $1.13(1.10-1.16)$ \\
\hline \multicolumn{2}{|l|}{ Educational level } \\
\hline$\leq$ Primary & $1.00($ ref $))$ \\
\hline Secondary & $0.39(0.21-0.73)$ \\
\hline University & $0.50(0.28-0.92)$ \\
\hline \multicolumn{2}{|l|}{ Health behaviors } \\
\hline \multicolumn{2}{|l|}{ Smoking } \\
\hline Never & 1.00 (ref) \\
\hline Former & $1.52(0.86-2.70)$ \\
\hline Current & $1.03(0.41-2.55)$ \\
\hline \multicolumn{2}{|l|}{ Alcohol consumption } \\
\hline Never & 1.00 (ref) \\
\hline Ex-drinker & $0.83(0.42-1.61)$ \\
\hline Current moderate drinker * & $0.53(0.31-0.91)$ \\
\hline Current heavy drinker * & $0.87(0.44-1.69)$ \\
\hline Time watching TV (hours/day) & $1.02(1.01-1.04)$ \\
\hline Physical activity (MET-hours/week) & $0.94(0.92-0.96)$ \\
\hline MEDAS (continuous) $\dagger$ & $0.90(0.80-0.99)$ \\
\hline Energy intake (per $100 \mathrm{kcal} /$ day) & $1.00(0.96-1.04)$ \\
\hline Abdominal obesity & $2.64(1.61-4.33)$ \\
\hline \multicolumn{2}{|l|}{ Morbidity } \\
\hline Cardiovascular disease $\neq$ & $1.57(0.77-3.17)$ \\
\hline Cancer & $1.56(0.43-5.60)$ \\
\hline Asthma or chronic bronchitis & $1.55(0.81-2.98)$ \\
\hline Osteoarthritis/arthritis & $2.09(1.32-3.32)$ \\
\hline Hip fracture & $1.80(0.54-6.03)$ \\
\hline \multicolumn{2}{|l|}{ Cardio-metabolic biomarkers } \\
\hline Hs-CRP (per mg/dl) & $1.48(1.23-1.80)$ \\
\hline Fibrinogen (per $\mathrm{g} / \mathrm{l})$ & $1.23(0.94-1.61)$ \\
\hline Leptin (per ng/dl, log-transformed) & $1.87(1.38-2.54)$ \\
\hline Systolic blood pressure (per $5 \mathrm{mmHg}$ ) & $1.01(0.96-1.06)$ \\
\hline HDL-cholesterol (per $5 \mathrm{mg} / \mathrm{dl})$ & $0.91(0.85-0.99)$ \\
\hline Triglycerides (per $5 \mathrm{mg} / \mathrm{l}$ ) & $1.04(1.02-1.05)$ \\
\hline LDL-cholesterol (per $5 \mathrm{mg} / \mathrm{dl})$ & $0.92(0.89-0.96)$ \\
\hline HbAlc (per $1 \%$ unit) & $1.48(1.20-1.81)$ \\
\hline \multicolumn{2}{|l|}{ Treatment of diabetes and of cardiovascular risk factors } \\
\hline Diabetes nutritional therapy (among diabetic participants) & $0.82(0.40-1.71)$ \\
\hline Oral antidiabetic drugs (among diabetic participants) & $1.59(0.77-3.29)$ \\
\hline Insulin (among diabetic participants) & $1.60(0.62-4.11)$ \\
\hline Antihypertensive drugs (among hypertensive participants) & $1.25(0.79-1.97)$ \\
\hline Lipid-lowering drugs (among hyperlipidemic participants) & $0.93(0.58-1.48)$ \\
\hline \multirow{2}{*}{\multicolumn{2}{|c|}{ * The threshold between moderate and heavy drinking is $\geq 20 \mathrm{~g} /$ day in men and $\geq 10 \mathrm{~g} /$ day in women }} \\
\hline & \\
\hline \multicolumn{2}{|l|}{ ‡ Ischemic heart disease, stroke or heart failure } \\
\hline HSCPD. Hirb-cencitivity $C$ regetive protain. H & \\
\hline
\end{tabular}


Table 3. Odds ratios ( $95 \%$ confidence interval) of frailty in diabetic versus non-diabetic subjects with progressive adjustment for possible mechanism of the association

\begin{tabular}{|c|c|}
\hline & $\begin{array}{l}\text { Odds ratio } \\
(95 \% \text { confidence } \\
\text { interval })\end{array}$ \\
\hline Adjusted for age, sex, and educational level (basic model) & $2.18(1.42-3.37)$ \\
\hline \multicolumn{2}{|l|}{ Additionally adjusted for health behaviors and morbidity } \\
\hline $\begin{array}{l}\text { Basic model and lifestyle factors (smoking, alcohol consumption, time } \\
\text { spent watching TV, recreational activity, MEDAS, energy intake) }\end{array}$ & $2.01(1.28-3.16)$ \\
\hline Basic model and abdominal obesity & $1.96(1.26-3.03)$ \\
\hline Basic model and lifestyle factors and abdominal obesity (model 1) & $1.83(1.16-2.90)$ \\
\hline \multicolumn{2}{|l|}{ Additionally adjusted for morbidity } \\
\hline $\begin{array}{l}\text { Model } 1 \text { and cardiovascular disease (heart disease, stroke, heart failure), } \\
\text { cancer, chronic respiratory disease, arthritis, osteoarthritis, fracture } \\
\text { (model 2) }\end{array}$ & $1.76(1.10-2.82)$ \\
\hline \multicolumn{2}{|l|}{ Additionally adjusted for cardio-metabolic biomarkers } \\
\hline Model 2 and HS-CRP & $1.71(1.06-2.76)$ \\
\hline Model 2 and fibrinogen & $1.75(1.09-2.80)$ \\
\hline Model 2 and leptin & $1.80(1.12-2.88)$ \\
\hline Model 2 and systolic blood pressure & $1.79(1.11-2.87)$ \\
\hline Model 2 and HDL-cholesterol & $1.64(1.00-2.69)$ \\
\hline Model 2 and HDL-cholesterol, triglycerides and LDL-cholesterol & $1.47(0.89-2.43)$ \\
\hline Model 2 and $\mathrm{HbA} 1 \mathrm{c}$ & $1.51(0.83-2.74)$ \\
\hline Model 2 and all the above mediators (model 3) & $1.32(0.70-2.49)$ \\
\hline \multicolumn{2}{|l|}{$\begin{array}{l}\text { Additionally adjusted for treatment of diabetes and of cardiovascular } \\
\text { risk factors }\end{array}$} \\
\hline Model 3 and diabetes nutritional therapy & $1.64(0.77-3.49)$ \\
\hline Model 3 and with oral antidiabetic drugs & $1.01(0.46-2.20)$ \\
\hline Model 3 and insulin & $1.29(0.68-2.45)$ \\
\hline $\begin{array}{l}\text { Model } 3 \text { and treatment with nutritional therapy, oral antidiabetics and/or } \\
\text { insulin }\end{array}$ & $1.28(0.57-2.91)$ \\
\hline Model 3 and antihypertensive drug treatment & $1.35(0.72-2.55)$ \\
\hline Model 3 and lipid-lowering drug treatment & $1.35(0.72-2.55)$ \\
\hline Model 3 and all the above treatments (model 4) & $1.32(0.58-2.98)$ \\
\hline
\end{tabular}

Analyses were based on 76 frailty cases among 1404 non-diabetic subjects and 39 frailty cases among 346 diabetic subjects

Hs-CRP: High-sensitivity C-reactive protein; HBA1c: Glycated hemoglobin. 
Table 4: Odds ratios (95\% confidence interval) of each frailty criterion in diabetic versus nondiabetic subjects with progressive adjustment for possible mechanism of the association.

\begin{tabular}{lc}
\hline & $\begin{array}{c}\text { Odds ratio } \\
\text { confidence interval) }\end{array}$ \\
\hline Exhaustion & \\
Model 1 & $0.97(0.59-1.60)$ \\
Model 2 & $0.95(0.58-1.58)$ \\
Model 3 & $0.99(0.53-1.85)$ \\
Model 4 & $0.60(0.24-1.51)$ \\
Low physical activity & \\
Model 1 & \\
Model 2 & \\
Model 3 & $0.76(0.48-1.20)$ \\
Model 4 & $0.76(0.48-1.20)$ \\
Slow walking speed & $0.69(0.39-1.20)$ \\
Model 1 & $0.66(0.31-1.41)$ \\
Model 2 & \\
Model 3 & \\
Model 4 & $\mathbf{1 . 4 9 ( 1 . 0 0 - 2 . 2 3 )}$ \\
Weight loss & $\mathbf{1 . 5 5}(\mathbf{1 . 0 3 - 2 . 3 3 )}$ \\
Model 1 & $1.34(0.81-2.22)$ \\
Model 2 & $1.43(0.74-2.76)$ \\
Model 3 & \\
Model 4 & \\
Weakness & \\
Model 1 & \\
Model 2 3 & $\mathbf{1 . 6 7}(\mathbf{1 . 0 0 - 2 . 7 7 )}$ \\
Model 4 & $1.60(0.95-2.69)$ \\
& $1.27(0.67-2.44)$ \\
& $1.93(0.91-4.08)$ \\
& \\
\hline
\end{tabular}

Analyses were based on the following cases (events/total in non-diabetic and diabetic individuals: Exhaustion (103/1158 and 23/251), low-physical activity (155/1158 and 28/251), slow walking speed (125/1150 and 40/249), weight loss (72/1148 and 24/249) and weakness (332/1157 and 87/250).

Model 1 is adjusted for age, sex, educational level, lifestyle factors and abdominal adiposity; Model 2 is further adjusted for self-reported morbidity; Model 3 is further adjusted for cardio-metabolic biomarkers; and model 4 is further adjusted for treatment of diabetes and of cardiovascular risk factors. 
Conflict-of-interest: The authors have nothing to disclose

Financial support: Baseline data collection was funded by Sanofi-Aventis. Data collection during follow-up was funded by the Spanish Government FIS grants 09/1626 and 09/0104 (Instituto de Salud Carlos III). Funding specific for this analysis was obtained from the Spanish Government FIS grant 12/1166 (Instituto de Salud Carlos III) and the FP7-HEALTH-2012-Proposal No: 305483-2 (FRAILOMIC Initiative). Funders had no role in data analyses, preparation of the manuscript, or in the decision to submit it for publication. 


\section{REFERENCES}

1. IDF Diabetes Atlas. 6th edition. Brussels: International Diabetes Federation 2013. Available at: http://www.idf.org/sites/default/files/EN 6E Atlas Full 0.pdf. 2014.

2. IHME (Institute for Health Metrics and Evaluation). Global Burden of Disease Arrow Diagram. Available at: http://vizhub.healthdata.org/irank/arrow.php. 2015.

3. McCrimmon RJ, Ryan CM, Frier BM: Diabetes and cognitive dysfunction. Lancet 379:2291-2299, 2012

4. Wong E, Backholer K, Gearon E, Harding J, Freak-Poli R, Stevenson C, Peeters A: Diabetes and risk of physical disability in adults: a systematic review and meta-analysis. Lancet Diabetes Endocrinol 1:106-114, 2013

5. Sinclair A, Dunning T, Rodriguez-Manas L: Diabetes in older people: new insights and remaining challenges. Lancet Diabetes Endocrinol 2014

6. Clegg A, Young J, lliffe S, Rikkert MO, Rockwood K: Frailty in elderly people. Lancet 381:752-762, 2013

7. Lee JS, Auyeung TW, Leung J, Kwok T, Leung PC, Woo J: Physical frailty in older adults is associated with metabolic and atherosclerotic risk factors and cognitive impairment independent of muscle mass. J Nutr Health Aging 15:857-862, 2011

8. Ottenbacher KJ, Graham JE, Al SS, Raji M, Samper-Ternent R, Ostir GV, Markides KS: Mexican Americans and frailty: findings from the Hispanic established populations epidemiologic studies of the elderly. Am J Public Health 99:673-679, 2009

9. Bouillon K, Kivimaki M, Hamer M, Shipley MJ, Akbaraly TN, Tabak A, Singh-Manoux A, Batty GD: Diabetes risk factors, diabetes risk algorithms, and the prediction of future frailty: the Whitehall II prospective cohort study. J Am Med Dir Assoc 14:851-856, 2013

10. Woods NF, LaCroix AZ, Gray SL, Aragaki A, Cochrane BB, Brunner RL, Masaki K, Murray A, Newman AB: Frailty: emergence and consequences in women aged 65 and older in the Women's Health Initiative Observational Study. J Am Geriatr Soc 53:1321-1330, 2005

11. Roschelle A, Heuberger RD: The frailty syndrome: A comprehensive review. J Nutr Gerontol Geriatr 30:314-368, 2011

12. Kendall DM, Bergenstal RM: Comprehensive management of patients with type 2 diabetes: establishing priorities of care. Am J Manag Care 7:S327-S343, 2001

13. Rodriguez-Artalejo F, Graciani A, Guallar-Castillon P, Leon-Munoz LM, Zuluaga MC, Lopez-Garcia E, Gutierrez-Fisac JL, Taboada JM, Aguilera MT, Regidor E, Villar-Alvarez F, Banegas JR: [Rationale and methods of the study on nutrition and cardiovascular risk in Spain (ENRICA)]. Rev Esp Cardiol 64:876-882, 2011

14. Fried LP, Tangen CM, Walston J, Newman AB, Hirsch C, Gottdiener J, Seeman T, Tracy R, Kop WJ, Burke G, McBurnie MA: Frailty in older adults: evidence for a phenotype. J Gerontol A Biol Sci Med Sci 56:M146-M156, 2001 
15. Pols MA, Peeters PH, Ocke MC, Slimani N, Bueno-de-Mesquita HB, Collette HJ: Estimation of reproducibility and relative validity of the questions included in the EPIC Physical Activity Questionnaire. Int J Epidemiol 26 Suppl 1:S181-S189, 1997

16. Martinez-Gonzalez MA, Lopez-Fontana C, Varo JJ, Sanchez-Villegas A, Martinez JA: Validation of the Spanish version of the physical activity questionnaire used in the Nurses' Health Study and the Health Professionals' Follow-up Study. Public Health Nutr 8:920-927, 2005

17. Relative validity and reproducibility of a diet history questionnaire in Spain. I. Foods. EPIC Group of Spain. European Prospective Investigation into Cancer and Nutrition. Int J Epidemiol 26 Suppl 1:S91-S99, 1997

18. Schroder H, Fito M, Estruch R, Martinez-Gonzalez MA, Corella D, Salas-Salvado J, Lamuela-Raventos R, Ros E, Salaverria I, Fiol M, Lapetra J, Vinyoles E, Gomez-Gracia E, Lahoz C, Serra-Majem L, Pinto X, Ruiz-Gutierrez V, Covas MI: A short screener is valid for assessing Mediterranean diet adherence among older Spanish men and women. J Nutr 141:1140-1145, 2011

19. Stamler J, Vaccaro O, Neaton JD, Wentworth D: Diabetes, other risk factors, and 12-yr cardiovascular mortality for men screened in the Multiple Risk Factor Intervention Trial. Diabetes Care 16:434-444, 1993

20. Zhang Y, Hu G, Yuan Z, Chen L: Glycosylated hemoglobin in relationship to cardiovascular outcomes and death in patients with type 2 diabetes: a systematic review and metaanalysis. PLoS One 7:e42551, 2012

21. Selvin E, Marinopoulos S, Berkenblit G, Rami T, Brancati FL, Powe NR, Golden SH: Metaanalysis: glycosylated hemoglobin and cardiovascular disease in diabetes mellitus. Ann Intern Med 141:421-431, 2004

22. Li H, Manwani B, Leng SX: Frailty, inflammation, and immunity. Aging Dis 2:466-473, 2011

23. Park SW, Goodpaster BH, Strotmeyer ES, Kuller LH, Broudeau R, Kammerer C, de RN, Harris TB, Schwartz AV, Tylavsky FA, Cho YW, Newman AB: Accelerated loss of skeletal muscle strength in older adults with type 2 diabetes: the health, aging, and body composition study. Diabetes Care 30:1507-1512, 2007

24. Leenders M, Verdijk LB, van der Hoeven L, Adam JJ, van KJ, Nilwik R, van Loon LJ: Patients with type 2 diabetes show a greater decline in muscle mass, muscle strength, and functional capacity with aging. J Am Med Dir Assoc 14:585-592, 2013

25. Park SW, Goodpaster BH, Strotmeyer ES, de RN, Harris TB, Schwartz AV, Tylavsky FA, Newman $A B$ : Decreased muscle strength and quality in older adults with type 2 diabetes: the health, aging, and body composition study. Diabetes 55:1813-1818, 2006

26. Blaum CS, Xue QL, Tian J, Semba RD, Fried LP, Walston J: Is hyperglycemia associated with frailty status in older women? J Am Geriatr Soc 57:840-847, 2009

27. Pariser G, Hager K, Gillette P, Golemboski K, Jackson K: Active steps for diabetes: a community-campus partnership addressing frailty and diabetes. Diabetes Educ 40:60-67, 2014 
28. Brescianini S, Maggi S, Farchi G, Mariotti S, Di CA, Baldereschi M, Inzitari D: Low total cholesterol and increased risk of dying: are low levels clinical warning signs in the elderly? Results from the Italian Longitudinal Study on Aging. J Am Geriatr Soc 51:991996, 2003

29. Schupf N, Costa R, Luchsinger J, Tang MX, Lee JH, Mayeux R: Relationship between plasma lipids and all-cause mortality in nondemented elderly. J Am Geriatr Soc 53:219226, 2005

30. Schalk BW, Visser M, Deeg DJ, Bouter LM: Lower levels of serum albumin and total cholesterol and future decline in functional performance in older persons: the Longitudinal Aging Study Amsterdam. Age Ageing 33:266-272, 2004

31. Carriere I, Peres K, Ancelin ML, Gourlet V, Berr C, Barberger-Gateau P, Bouillon K, Kivimaki $\mathrm{M}$, Ritchie $\mathrm{K}$, Akbaraly $\mathrm{T}$ : Metabolic syndrome and disability: findings from the prospective three-city study. J Gerontol A Biol Sci Med Sci 69:79-86, 2014

32. Landi F, Russo A, Cesari M, Pahor M, Bernabei R, Onder G: HDL-cholesterol and physical performance: results from the ageing and longevity study in the sirente geographic area (ilSIRENTE Study). Age Ageing 36:514-520, 2007

33. Landi F, Russo A, Pahor M, Capoluongo E, Liperoti R, Cesari M, Bernabei R, Onder G: Serum high-density lipoprotein cholesterol levels and mortality in frail, community-living elderly. Gerontology 54:71-78, 2008

34. Franz MJ, Boucher JL, Evert AB: Evidence-based diabetes nutrition therapy recommendations are effective: the key is individualization. Diabetes Metab Syndr Obes 7:65-72, 2014

35. Al-Shookri A, Khor GL, Chan YM, Loke SC, Al-Maskari M: Effectiveness of medical nutrition treatment delivered by dietitians on glycaemic outcomes and lipid profiles of Arab, Omani patients with Type 2 diabetes. Diabet Med 29:236-244, 2012

36. Barakatun Nisak MY, Ruzita AT, Norimah AK, Kamaruddin NA: Medical nutrition therapy administered by a dietitian yields favourable diabetes outcomes in individual with type 2 diabetes mellitus. Med J Malaysia 68:18-23, 2013

37. Rendel M: Advances in diabetes for the millennium: nutritional therapy of type 2 diabetes. MedGenMed 6:10, 2004

38. Cetrone M, Mele A, Tricarico D: Effects of the antidiabetic drugs on the age-related atrophy and sarcopenia associated with diabetes type II. Curr Diabetes Rev 10:231-237, 2014

39. Lee CG, Schwartz AV, Yaffe K, Hillier TA, LeBlanc ES, Cawthon PM: Changes in physical performance in older women according to presence and treatment of diabetes mellitus. J Am Geriatr Soc 61:1872-1878, 2013

40. Visser M, Goodpaster BH, Kritchevsky SB, Newman AB, Nevitt M, Rubin SM, Simonsick EM, Harris TB: Muscle mass, muscle strength, and muscle fat infiltration as predictors of incident mobility limitations in well-functioning older persons. J Gerontol A Biol Sci Med Sci 60:324-333, 2005 
41. Cawthon PM, Fox KM, Gandra SR, Delmonico MJ, Chiou CF, Anthony MS, Sewall A, Goodpaster B, Satterfield S, Cummings SR, Harris TB: Do muscle mass, muscle density, strength, and physical function similarly influence risk of hospitalization in older adults? J Am Geriatr Soc 57:1411-1419, 2009

42. Newman AB, Kupelian V, Visser M, Simonsick EM, Goodpaster BH, Kritchevsky SB, Tylavsky FA, Rubin SM, Harris TB: Strength, but not muscle mass, is associated with mortality in the health, aging and body composition study cohort. J Gerontol A Biol Sci Med Sci 61:72-77, 2006

43. Volpato S, Bianchi L, Lauretani F, Lauretani F, Bandinelli S, Guralnik JM, Zuliani G, Ferrucci $\mathrm{L}$ : Role of muscle mass and muscle quality in the association between diabetes and gait speed. Diabetes Care 35:1672-1679, 2012

44. Kalyani RR, Tra Y, Yeh HC, Egan JM, Ferrucci L, Brancati FL: Quadriceps strength, quadriceps power, and gait speed in older U.S. adults with diabetes mellitus: results from the National Health and Nutrition Examination Survey, 1999-2002. J Am Geriatr Soc 61:769-775, 2013

45. IJzerman TH, Schaper NC, Melai T, Meijer K, Willems PJ, Savelberg HH: Lower extremity muscle strength is reduced in people with type 2 diabetes, with and without polyneuropathy, and is associated with impaired mobility and reduced quality of life. Diabetes Res Clin Pract 95:345-351, 2012

46. Strotmeyer ES, de RN, Schwartz AV, Faulkner KA, Resnick HE, Goodpaster BH, Shorr RI, Vinik Al, Harris TB, Newman AB: The relationship of reduced peripheral nerve function and diabetes with physical performance in older white and black adults: the Health, Aging, and Body Composition (Health ABC) study. Diabetes Care 31:1767-1772, 2008

47. Jor'dan AJ, Manor B, Novak V: Slow gait speed - an indicator of lower cerebral vasoreactivity in type 2 diabetes mellitus. Front Aging Neurosci 6:135, 2014

48. Kelly C, Fleischer A, Yalla S, Grewal GS, Albright R, Berns D, Crews R, Najafi B: Fear of falling is prevalent in older adults with diabetes mellitus but is unrelated to level of neuropathy. J Am Podiatr Med Assoc 103:480-488, 2013

49. Lee JS, Kritchevsky SB, Tylavsky F, Harris T, Simonsick EM, Rubin SM, Newman AB: Weight change, weight change intention, and the incidence of mobility limitation in wellfunctioning community-dwelling older adults. J Gerontol A Biol Sci Med Sci 60:10071012,2005

50. Ritchie CS, Locher JL, Roth DL, McVie T, Sawyer P, Allman R: Unintentional weight loss predicts decline in activities of daily living function and life-space mobility over 4 years among community-dwelling older adults. J Gerontol A Biol Sci Med Sci 63:67-75, 2008

51. Wedick NM, Barrett-Connor E, Knoke JD, Wingard DL: The relationship between weight loss and all-cause mortality in older men and women with and without diabetes mellitus: the Rancho Bernardo study. J Am Geriatr Soc 50:1810-1815, 2002

52. Gregg EW, Gerzoff RB, Thompson TJ, Williamson DF: Trying to lose weight, losing weight, and 9-year mortality in overweight U.S. adults with diabetes. Diabetes Care 27:657-662, 2004 
53. Graciani A, Rodriguez-Artalejo F, Navarro-Vidal B, Banegas JR: Glycemic control using individualized targets among diabetic patients in Spain: a population-based study. Rev Esp Cardiol (Engl Ed) 67:151-153, 2014

54. Lipska KJ, Ross JS, Miao Y, Shah ND, Lee SJ, Steinman MA: Potential overtreatment of diabetes mellitus in older adults with tight glycemic control. JAMA Intern Med 175:356362,2015 